\title{
New Perspectives on Music in Rehabilitation of Executive and Attention Functions
}

\author{
Yuko Koshimori^ and Michael H. Thaut \\ Music and Health Research Collaboratory, Faculty of Music, University of Toronto, Toronto, ON, Canada
}

\section{OPEN ACCESS}

Edited by:

Paul J. Colombo,

Tulane University, United States

Reviewed by:

Aline Moussard,

Centre de Recherche de l'Institut Universitaire de Gériatrie de Montréal

(CRIUGM), Canada

Alfredo Raglio,

Scientific Clinical Institutes Maugeri

IRCCS (ICS Maugeri), Italy

*Correspondence:

Yuko Koshimori

yuko.koshimori@mail.utoronto.ca

Specialty section:

This article was submitted to Auditory Cognitive Neuroscience,

a section of the journal

Frontiers in Neuroscience

Received: 03 June 2019

Accepted: 05 November 2019

Published: 19 November 2019

Citation:

Koshimori Y and Thaut MH (2019)

New Perspectives on Music

in Rehabilitation of Executive

and Attention Functions.

Front. Neurosci. 13:1245

doi: 10.3389/fnins.2019.01245
Modern music therapy, starting around the middle of the twentieth century was primarily conceived to promote emotional well-being and to facilitate social group association and integration. Therefore, it was rooted mostly in social science concepts. More recently, music as therapy began to move decidedly toward perspectives of neuroscience. This has been facilitated by the advent of neuroimaging techniques that help uncover the therapeutic mechanisms for non-musical goals in the brain processes underlying music perception, cognition, and production. In this paper, we focus on executive function (EF) and attentional processes (AP) that are central for cognitive rehabilitation efforts. To this end, we summarize existing behavioral as well as neuroimaging and neurophysiological studies in musicians, non-musicians, and clinical populations. Musical improvisation and instrumental playing may have some potential for EF/AP stimulation and neurorehabilitation. However, more neuroimaging studies are needed to investigate the neural mechanisms for the active musical performance. Furthermore, more randomized clinical trials combined with neuroimaging techniques are warranted to demonstrate the specific efficacy and neuroplasticity induced by music-based interventions.

Keywords: executive function, attention processes, music neuroscience, music improvisation, Neurologic Music Therapy

\section{INTRODUCTION}

Brain and biomedical research involving music has shown that music is a highly structured auditory language engaging complex perception, cognition, and motor control in the brain (Peretz and Zatorre, 2005; Stewart et al., 2006; Alluri et al., 2011; Lee et al., 2011; Thaut et al., 2014) and has a distinct influence on the brain by stimulating complex cognitive (Leggieri et al., 2019), affective (Koelsch, 2014), and sensorimotor processes (Thaut et al., 2015; Crasta et al., 2018; Schaffert et al., 2019).

As musical sound patterns are dynamic, continuously unfold, and change in time, external auditory input may serve well for cognitive rehabilitation, in particular for the domains of executive function (EF) and attentional processes (AP) by helping to adapt to a changing environment. Music by its nature drives attention exogenously (Klein and Lawrence, 2011; Thaut and Gardiner, 2014). Furthermore, music is a multidimensional stimulus consisting of multiple acoustical elements (e.g., pitch, loudness, tempo, rhythms, timbre, melody, harmony) that exist in temporally patterned simultaneity and sequentiality to drive AP (Thaut and Gardiner, 2014). In addition, active music performance involves moment-to-moment feedback systems, which facilitates monitoring, adjusting, and updating (Gardiner and Thaut, 2014). Further, there is some evidence that musicians 
perform better on EF/AP (e.g., Hanna-Pladdy and Mackay, 2011) as well as there are prefrontal structural and functional differences between musicians and non-musicians (e.g., Fauvel et al., 2014; Groussard et al., 2014). Furthermore, research shows that active musical performance extensively modulates the prefrontal activity in musicians (Erkkinen and Berkowitz, 2018).

Therefore, this sensory language may effectively be used as a therapy to induce neuroplasticity in the brain affected by disorders, diseases, and injuries (Särkämö et al., 2014). With this neuroscientific knowledge, music may be able to yield more specific therapeutic and stimulation outcomes for targeted functions. In this paper, we first briefly summarize existing studies investigating the effects of formal musical training on the $\mathrm{EF} / \mathrm{AP}$ performance as well as on brain structure and function. Second, we discuss the neuroimaging/neurophysiological studies demonstrating the prefrontal neural correlates and neuroplasticity of active musical performance. Third, we present the body of literature in music-based interventions in healthy and clinical populations, focusing on the EF/AP findings. Finally, we present the future directions of research in this field to move forward the neuroscientific approach, which facilitates further advancement of music-based interventions for the EF/AP stimulation and rehabilitation.

\section{MUSIC TRAINING ON EF/AP AND BRAIN STRUCTURE AND FUNCTION IN MUSICIANS}

Some correlational and cross sectional studies have supported the beneficial effects of music training on EF/AP in older musicians (Hanna-Pladdy and Mackay, 2011; Hanna-pladdy and Gajewski, 2012; Liu et al., 2012; Amer et al., 2013; Moussard et al., 2016; Strong and Mast, 2019), younger musicians (Moradzadeh and Blumenthal, 2015; Okada and Slevc, 2018; Medina and Barraza, 2019), and musically trained children (Zuk et al., 2014). In addition, several studies demonstrated structural and functional changes associated with formal musical training in the prefrontal regions. For example, greater gray matter volume was associated with increasing musical practice in the multiple regions including supplementary motor area (SMA), superior/middle and medial frontal cortex, and insula (Groussard et al., 2014; James et al., 2014). Furthermore, resting-state functional connectivity revealed significantly greater connectivity density in the medial and lateral prefrontal regions as well as temporoparietal junction in musicians (Fauvel et al., 2014; Luo et al., 2014; Klein et al., 2016; Tanaka and Kirino, 2016).

A few studies investigated the brain activity during an EF task in musicians. One EEG study using a visual go/no-go task demonstrated that a group of 17 older musicians exhibited a significantly larger difference in the N2 amplitude (reflecting a conflict detect signal or inhibition of a prepotent response) in the central midline sites between go and no-go conditions compared to a group of 17 older non-musicians (Moussard et al., 2016). The music group also performed significantly better on the task, demonstrated with fewer no-go errors. Further, the music group showed a significant correlation between the N2 amplitude and the no-go task performance. However, there were no significant correlations between the N2 amplitude and any measures of musical background (age of first instruction, years of musical instruction, and hours of current practice). On the other hand, the measures of musical background, but not the task performance were significantly associated with the P3 amplitude that showed no significant group difference in amplitude but differential topography between groups (anterior shift in musicians). Two other studies demonstrated differential brain activities during EF tasks in younger musicians (Moreno et al., 2014) and musically trained children (Zuk et al., 2014) compared to non-musician groups without showing task performance differences between groups.

In summary, the cross-sectional or correlational studies in musicians have shed light on the potential benefit of formal musical training on EF/AP and brain changes in the prefrontal area. The task-based studies consistently demonstrated that individuals with formal musical training have differential brain activity during EF tasks in relative to those without it. However, these studies do not allow to determine the direct and causal effects of music training on EF/AP and brain changes. Furthermore, in these musician studies, the specific effects of different types of musical training on the EF/AP as well as on brain structure and function in the prefrontal area are still unclear.

\section{NEURAL MECHANISMS FOR ACTIVE MUSICAL PERFORMANCE}

Several neuroimaging/neurophysiological studies investigated the brain activity during active musical performance in musician. One study using near-infrared spectroscopy (NIRS) reported that piano playing engaged the frontal activity and playing more complex musical piece activated significantly wider frontal areas in musicians (Hashimoto and Okamoto, 2006). Furthermore, the brain activity during musical improvisation was investigated in amateur and professional musicians with varying experience of improvisation. These studies suggest that this creative musical activity extensively engages the brain activity in the prefrontal regions such as dorsal premotor area, pre-SMA, SMA, medial prefrontal cortex (mPFC), dorsolateral prefrontal cortex (DLPFC), anterior cingulate cortex (ACC), inferior frontal gyrus (IFG), and anterior insula (Brown et al., 2006; Pinho et al., 2014; Beaty, 2015; Erkkinen and Berkowitz, 2018; Loui, 2018). However, the directionality of improvisationinduced brain activity varied across the studies. The enhanced prefrontal brain activity and connectivity was interpreted as a reflection of goal-directed, top-down processing including motor planning, response selection, inhibition of competing stimuli, and conscious monitoring (Bengtsson, 2007; Villarreal et al., 2013). The dissociation of decreased lateral and increased medial prefrontal brain activity was interpreted as a reflection of a disconnection or disintegration of the lateral regions to suppress the top-down conscious control to generate spontaneous ideas, engaging the medial prefrontal regions, which was observed in more experienced improvisers (Limb and Braun, 2008; 
Liu et al., 2012). Further, the decreased lateral prefrontal activity and functional connectivity was interpreted as indicating that experts could spare EF load for the highly automatized activity (de Manzano and Ullén, 2012; Pinho et al., 2014; Rosen et al., 2016). The observed activation or deactivation in the lateral prefrontal cortex may also depend on the degree of top-down control required by a given improvisation task (Berkowitz and Ansari, 2008; Pinho et al., 2014). For example, the deactivation in the fronto-parietal regions was greater as the freedom of improvisation task increased (Berkowitz and Ansari, 2008). On the other hand, the enhanced lateral prefrontal activity observed in experienced improvisers may be due to the constrains imposed on the improvisation task (Bengtsson, 2007). In addition, the existing studies demonstrated that musical improvisation induced greater brain activity in the prefrontal regions such as DLPFC compared to simple musical repetitive or patterned tasks (Berkowitz and Ansari, 2008; Villarreal et al., 2013) and reproducing music (de Manzano and Ullén, 2012).

In summary, piano playing and musical improvisation extensively engages the prefrontal activity. This is likely because these musical activities involve EF/AP (Hannon and Trainor, 2007; Beaty, 2015). In addition, musical improvisation induces greater activation of the prefrontal regions and functional connectivity in musicians with less experience of improvisation and compared to simple musical repetitive and patterned tasks. Therefore, these musical activities may have some potential for EP/AP stimulation and neurorehabilitation.

\section{MUSIC-BASED INTERVENTIONS FOR EF AND AP STIMULATION IN NON-MUSICIANS}

There are a few studies that have demonstrated benefits of learning to play a musical instrument on EF in older healthy nonmusicians. In one randomized control trial (RCT), 16 older adults received individualized piano lessons for 6 months (Bugos et al., 2007). They exhibited improvement on the Trail Making Test-B (TMT-B) over time during the period. This was not observed in a control group consisting of 15 older adults. In another study, a 4-month weekly group piano lessons designed and implemented by a professional music teacher and pianist resulted in improved performance on the Color-Word Stroop test in 13 older adults. This cognitive improvement was not observed in 16 older adults of the control group (Seinfeld et al., 2013). Another study further supported the benefit of group piano lessons on EF such as verbal flexibility and inhibition control in 24 older adults (Bugos, 2010). The EF improvement was significantly greater compared to a music appreciation group consisting of 22 older adults who had learned musical elements while listening to music. However, both music groups significantly improved the EF performance.

In addition, one EEG study with a pseudo-randomized design investigated the effects of music making on inhibition control and interference (Alain et al., 2019). Sixty healthy older non-musicians received either 3-month musical, visual art, or no training. The music-based intervention included music making using body percussion, and voice and non-pitched musical instruments, as well as learning basic music theory, and melody and harmony concepts by singing simple canons. It was provided by a professional music teacher. Transient differential neural activities were observed in the fronto-central sites in both intervention groups without showing improvement on the task performance.

In summary, learning to play the piano may have beneficial effects on EF in healthy older non-musicians. This may be because it is a complex process, requiring processes for the coordination of multiple sensory modalities, motor control, monitoring, working memory, inhibition, and attentional shifting (Hannon and Trainor, 2007; Seinfeld et al., 2013). However, the sample sizes of these studies are small with additional methodological limitations in some of the studies such as no randomization, no blind assessors, no active control group, and unknown intervention compliance.

\section{MUSIC-BASED INTERVENTIONS FOR EF AND AP NEUROREHABILITATION IN CLINICAL POPULATIONS}

Several clinical studies incorporated musical improvisation and instrument playing as their intervention techniques. A few pilot studies investigated the effects of Neurologic Music Therapy (NMT) training for EF/AP. It is guided by a NMT-certified music therapist and is based on group improvisation projects with a special emphasis on attention switching that requires participants to musically respond to specific musical cues (Gardiner and Thaut, 2014; Thaut and Gardiner, 2014). In a pseudo-experimental design (due to institutional constraints of U.S. Veterans Administration), 31 persons with acquired brain dysfunction received one 30-min NMT training session (Thaut et al., 2009). A paired $t$-test analysis showed that the music group significantly improved cognitive flexibility assessed on TMT-B with a large effect size $(d=1.21)$, but not memory functions. A control group $(N=23)$ without any intervention did not show any change on the test. In addition, the music group significantly increased their confidence in the EF skill. Other NMT studies included children/adolescents with neurodevelopmental disorders such as Attention Deficit Hyperactivity Disorder (Abrahams and van Dooren, 2018) and Autism Spectrum Disorder (Pasiali et al., 2014). In both studies, the interventions consisted of a 45-min weekly training session over a period of 6 weeks. Both studies reported some improvement on AP. However, both studies included small sample sizes (2 participants in the NMT group in the former study and 9 participants with varying severity in the latter study). Due to the methodological limitations, these NMT preliminary findings need to replicate in RCTs with large sample sizes.

In addition, one RCT included 35 older adults with Mini Mental State Examination scores $\geq 18$ and employed a 12 biweek group music cognitive training (Biasutti and Mangiacotti, 2018). The training was delivered by a specialist with music and neuropsychology background and consisted of voice and instrumental improvisation, which was created based on the 
framework of different music-based interventions including NMT (Thaut, 2005). Repeated ANOVA showed that the music group $(N=18)$ showed significant improvement in mental flexibility assessed on verbal fluency and showed a trend toward significance in selective attention assessed on Attentional Matrices test compared to an active control group (gymnastic activities; $N=17$ ).

In another RCT, 28 chronic stroke patients without extensive musical experience received either $30-\mathrm{h}$ of music-supported therapy (MST) or conventional physical training over a 10-week period (Fujioka et al., 2018). The MST protocol included mapping functional movements on playing musical instruments, which was based on the NMT technique (Thaut, 2005), as well as music-making with a therapist. The music group showed significant improvement in cognitive flexibility measured by TMT-B in the mid-intervention whereas the active control group showed the improvement post-intervention.

In one RCT combined with functional near-infrared spectroscopy (fNIRS), 39 persons with mild cognitive impairment were assigned either to an instructor-guided 12-week movement music therapy (MMT) or a comparable active non-musical control therapy (Shimizu et al., 2018). MMT included light exercises synchronized to the background music and playing a percussion instrument accompanied with familiar songs. The MMT group showed enhanced mPFC activity and increased functional connectivity within the prefrontal area compared to the control group. ANOVA did not show a significant group effect on the Frontal Assessment Battery score. This is likely because both groups showed some improvement post-intervention in which the MMT group showed a significant improvement while the active control group showed a trend toward significance, shown using a paired $t$-test. A major limitation of this study was that the number of participants in each group was imbalanced (MMT: $N=30$ vs. control: $N=9$ ).

There are also RCTs that demonstrated the beneficial effects of other music-based interventions on EF/AP. For example, a 12-week singing (four different familiar songs) intervention guided by a professional choir instructor led to a significant improvement on inhibitory control in 31 persons with mild Alzheimer's disease (AD; Pongan et al., 2017). This positive effect was also observed in the active control group (painting) of 28 mild AD participants. Another RCT employed Sound Training for Attention and Memory in Dementia (STAMDem; Ceccato et al., 2012). STAM-Dem is a 12-week musicbased manualized protocol delivered by a music therapist in which the participants perform specific movements to instructed sound stimuli that requires selective attention. It consists of multiple phases with increasing difficulty. Test score changes on selective attention were significantly greater (improved test score) in the music group of 27 participants compared to the control group of 23 participants who received standard care. In addition, 1-h daily listening to participant-selected favorite music guided by a music therapist for 2 months significantly improved AP recovery in 17 persons with acute post-stroke compared to both active and passive control groups of 19 and 17 participants, respectively (Särkämö et al., 2008). Furthermore, the AP improvement was also associated with a significant increase in the prefrontal gray matter volume in those with left hemisphere stroke (Särkämö et al., 2014).

In summary, clinical studies showed some potential of active music-based interventions such as musical improvisation and instrument playing-based activities to enhance EF/AP. However, their specific effectiveness is still unclear as active control interventions are often effective as well. In addition, there is a paucity of research literature with various clinical conditions. With few neuroimaging studies, it is still to be determined whether the music-based interventions have engaged executive control areas or whether neuroplasticity had occurred in those areas. Further, some studies show critical limitations in the research design such as a small sample size, no randomization, no active control condition, no follow-up assessment, no analysis of a group $\times$ time interaction effect, and no trained therapist.

\section{FUTURE DIRECTIONS AND PERSPECTIVES FOR EF/AP STIMULATION AND NEUROREHABILITATION}

The current paper summarizes the effects of formal musical training, active musical performance, and music-based interventions on EF/AP and associated brain activity in musicians, non-musicians, and clinical populations to present the future directions and perspectives of music-based interventions for the EF/AP stimulation and neurorehabilitation. Active musical performance such as piano playing and musical improvisation engages the prefrontal activity and some intervention studies showed the potential of these musical activities for a basis of the EF/AP stimulation and neurorehabilitation techniques. One study showed that enhanced inhibition control was associated with the extent of involvement of the musical activity in the motor system via the cognitive control system in musicians (Slater et al., 2017), suggesting that instrument playing may be more effective to enhance inhibition control compared to singing, for example. However, neuroimaging studies are needed to uncover the neural mechanisms for piano playing and musical improvisation in non-musicians and to demonstrate the direct link between these musical activities and the EF/AP performance. It is also useful for such studies to contrast images between musical activities and the resting state activity as well as between the two musical activities to show what areas are involved in each musical activity and how similar or different in the brain activity induced by these musical activities. Further, more RCTs with a rigorous design are warranted to replicate the findings. In addition, it is important to address whether these interventions are feasible and effective for different age groups and clinical populations with different clinical characteristics (e.g., severity, acute vs. chronic).

Furthermore, it is crucial to design music-based interventions that can tap into targeted processing components of EF/AP. Some of the clinical studies specifically targeted an enhancement of cognitive flexibility and selective attention, utilizing acoustic elements of music as cues or target stimuli (Thaut et al., 2009; 
Ceccato et al., 2012). However, as music is tightly connected to the attention system (Särkämö et al., 2008), it can temporally enhance cognitive performance associated with some of the AP as well as learning and memory via increased arousal (Hommel et al., 1990; Thaut et al., 2005; Thompson et al., 2006; Särkämö et al., 2008). Therefore, future RCTs should include music listening as an active control condition to determine the specific efficacy of active music-based interventions above the music-induced arousal effect on EF/AP with follow-up assessments.

Lastly, neuroimaging techniques should be combined to determine whether music-based interventions engage the brain activity of the executive control regions and networks as well as whether neuroplasticity has occurred in the expected regions and networks following the interventions. These studies are also useful to determine whether the observed patterns of hyper- or hypo-activations are due to compensation, neural efficiency, or attempted compensation (Grady, 2012). Such research studies can help growth in this domain, shedding

\section{REFERENCES}

Abrahams, T. P., and van Dooren, J. C. (2018). The arts in psychotherapy musical attention control training (MACT) in secure residential youth care: a randomised controlled pilot study. Arts. Psychother. 57, 80-87. doi: 10.1016/j. aip.2017.10.008

Alain, C., Moussard, A., Singer, J., Lee, Y., Bidelman, G. M., and Francisco, G. (2019). Music and visual art training modulate brain activity in older adults. Front. Neurosci. 13:1-15. doi: 10.3389/fnins.2019.00182

Alluri, V., Toiviainen, P., Jääskeläinen, I. P., Glerean, E., Sams, M., and Brattico, E. (2011). Large-scale brain networks emerge from dynamic processing of musical timbre, key and rhythm. NeuroImage 59, 3677-3689. doi: 10.1016/j.neuroimage. 2011.11.019

Amer, T., Kalender, B., Hasher, L., Trehub, S. E., and Wong, Y. (2013). Do older professional musicians have cognitive advantages? PLoS One 8:e71630. doi: 10.1371/journal.pone.0071630

Beaty, R. E. (2015). The neuroscience of musical improvisation. Neurosci. Biobehav. Rev. 51, 108-117. doi: 10.1016/j.neubiorev.2015.01.004

Bengtsson, S. L. (2007). Cortical regions involved in the generation of musical structures during improvisation in pianists. J. Cogn. Neurosci. 19, 830-842. doi: 10.1162/jocn.2007.19.5.830

Berkowitz, A. L., and Ansari, D. (2008). Generation of novel motor sequences: the neural correlates of musical improvisation. 41, 535-543. doi: 10.1016/j. neuroimage.2008.02.028

Biasutti, M., and Mangiacotti, A. (2018). Assessing a cognitive music training for older participants: a randomised controlled trial. Int. J. Geriatirc Psychiatry Geriatirc. Psychiatry 33, 271-278. doi: 10.1002/gps.4721

Brown, S., Martinez, M., and Parsons, L. (2006). Music and language side by side in the brain : a PET study of the generation of melodies and sentences. Eur. J. Neurosci. 23, 2791-2803. doi: 10.1111/j.1460-9568.2006.04785.x

Bugos, J. A. (2010). The benefits of music instruction on processing speed, verbal fluency, and cognitive control in aging. Music Educ. Res. Int. 4, 1-9.

Bugos, J. A., Perlstein, W. M., Mccrae, C. S., Brophy, T. S., Bedenbaugh, P. H., Perlstein, W. M., et al. (2007). Individualized piano instruction enhances executive functioning and working memory in older adults. Aging Ment. Heal. 11, 464-471. doi: 10.1080/13607860601086504

Ceccato, E., Vigato, G., Bonetto, C., Bevilacqua, A., Pizziolo, P., Crociani, S., et al. (2012). STAM Protocol in dementia: a and controlled trial. Am. J. Alzheimers Dis. Other Demen. 27, 301-310. doi: 10.1177/1533317512452038

Crasta, J. E., Thaut, M. H., Anderson, C. W., Davies, P. L., and Gavin, W. J. (2018). Auditory priming improves neural synchronization in auditory-motor entrainment. Neuropsychologia 117, 102-112. doi: 10.1016/j.neuropsychologia. 2018.05.017 an increasing light on the neural mechanisms of how musicbased intervention techniques can tap into higher cognition to facilitate maintenance, enhancement, as well as recovery of cognitive functions.

\section{AUTHOR CONTRIBUTIONS}

YK conceived the concept of the manuscript. YK wrote the first draft of the manuscript and MT wrote the sections of the draft. Both authors contributed to manuscript revision, and read and approved the submitted version of the manuscript.

\section{FUNDING}

This research was supported by the Canada Research Chair Program.

de Manzano, Ö, and Ullén, F. (2012). Goal-independent mechanisms for free response generation: creative and pseudo-random performance share neural substrates. Neuroimage 59, 772-780. doi: 10.1016/j.neuroimage.2011.07.016

Erkkinen, M. G., and Berkowitz, A. L. (2018). "Brain research in music improvisation," in The Oxford Handbook of Music and the Brain, eds D. A. Hodges, and M. H. Thaut (UK: Oxford University Press), 1-38. doi: 10.1093/ oxfordhb/9780198804123.013.20

Fauvel, B., Groussard, M., Chételat, G., Fouquet, M., Landeau, B., Eustache, F., et al. (2014). NeuroImage morphological brain plasticity induced by musical expertise is accompanied by modulation of functional connectivity at rest. Neuroimage 90, 179-188. doi: 10.1016/j.neuroimage.2013.12.065

Fujioka, T., Dawson, D. R., Wright, R., Honjo, K., Chen, J. L., Chen, J. J., et al. (2018). The effects of music-supported therapy on motor, cognitive, and psychosocial functions in chronic stroke. Ann. N. Y. Acad. Sci. doi: 10.1111/ nyas.13706 [Epub ahead of print].

Gardiner, J. C., and Thaut, M. (2014). "Musical executive function training (MEET)," in Handbook of Neurologic Music Therapy, eds M. H. Thaut, and V. Hoemberg, (Oxford: Oxford University Press), 279-293.

Grady, C. (2012). The cognitive neuroscience of ageing. Nat. Rev. Neurosci. 13, 491-505. doi: 10.1038/nrn3256

Groussard, M., Viader, F., Landeau, B., and Desgranges, B. (2014). Brain and cognition the effects of musical practice on structural plasticity: the dynamics of grey matter changes. Brain Cogn. 90, 174-180. doi: 10.1016/j.bandc.2014.06.013

Hanna-pladdy, B., and Gajewski, B. (2012). Recent and past musical activity predicts cognitive aging variability: direct comparison with general lifestyle activities. Front. Hum. Neurosci. 6:198. doi: 10.3389/fnhum.2012.00198

Hanna-Pladdy, B., and Mackay, A. (2011). The relation between instrumental musical activity and cognitive aging. Neuropsychology 25, 378-386.

Hannon, E. E., and Trainor, L. J. (2007). Music acquisition: effects of enculturation and formal training on development. Trends Cogn. Sci. 11, 466-472. doi: 10. 1016/j.tics.2007.08.008

Hashimoto, K., and Okamoto, T. (2006). Examination by near-infrared spectroscopy for evaluation of piano performance as a frontal. Eur. Neurol. 55, 16-21. doi: 10.1159/000091138

Hommel, M., Peres, B., Pollak, P., Memin, B., Besson, G., Gaio, J. M., et al. (1990). Effects of passive tactile and auditory stimulion left visual neglect. Arch. Neurol. 47, 573-576. doi: 10.1001/archneur.1990.00530050097018

James, C. E., Oechslin, M. S., Hauert, C. A., and Van De Ville, D. (2014). Musical training intensity yields opposite effects on grey matter density in cognitive versus sensorimotor networks. Brain Struct. Funct. 210, 353-366. doi: 10.1007/ s00429-013-0504-z

Klein, C., Liem, F., Elmer, S., and Lutz, J. (2016). The "Silent" imprint of musical training. Hum. Brain Mapp. 37, 536-546. doi: 10.1002/hbm.23045 
Klein, R., and Lawrence, M. (2011). “On the modes and domains of attention," in Cognitive Neuroscience of Attention, ed. M. I. Posner, (New York, NY: Guilford), 11-28.

Koelsch, S. (2014). Brain correlates of music-evoked emotions. Nat. Rev. Neurosci. 15, 170-180. doi: 10.1038/nrn3666

Lee, Y., Janata, P., Frost, C., Hanke, M., and Granger, R. (2011). Investigation of melodic contour processing in the brain using multivariate pattern-based fMRI. NeuroImage 57, 293-300. doi: 10.1016/j.neuroimage.2011.02.006

Leggieri, M., Thaut, M. H., Fornazzari, L., Schweizer, T. A., Barfett, J., Munoz, D. G., et al. (2019). Music intervention approaches for alzheimer's disease: a review of the literature. Front. Neurosci. 13:132. doi: 10.3389/fnins.2019.00132

Limb, C., and Braun, A. (2008). Neural substrates of spontaneous musical performance: a fMRI study of jazz improvisation. PLoS One 3:e1679. doi: 10. 1371/journal.pone.0001679

Liu, S., Chow, H. M., Xu, Y., Erkkinen, M. G., Swett, K. E., Eagle, M. W., et al. (2012). Neural correlates of lyrical improvisation: an fMRI study of freestyle rap. Sci. Rep. 2, 834. doi: 10.1038/srep00834

Loui, P. (2018). Rapid and flexible creativity in musical improvisation: review and a model. Ann. N. Y. Acad. Sci. doi: 10.1111/nyas.13628 [Epub ahead of print].

Luo, C., Tu, S., Peng, Y., Gao, S., Li, J., Dong, L., et al. (2014). Long-term effects of musical training and functional plasticity in salience system. Neural Plast. 2014, 13. doi: $10.1155 / 2014 / 180138$

Medina, D., and Barraza, P. (2019). Efficiency of attentional networks in musicians and non-musicians. Heliyon 5:e01315. doi: 10.1016/j.heliyon.2019.e01315

Moradzadeh, L., and Blumenthal, G. (2015). Musical training, bilingualism, and executive function: a closer look at task switching and dual-task performance. Cogn. Sci. 39, 992-1020. doi: 10.1111/cogs.12183

Moreno, S., Wodniecka, Z., Tays, W., Alain, C., and Bialystok, E. (2014). Inhibitory control in bilinguals and musicians: event related potential (ERP) evidence for experience-specific effects. PLoS One 9:e94169. doi: 10.1371/journal.pone. 0094169

Moussard, A., Bermudez, P., Alain, C., Tays, W., and Moreno, S. (2016). Life-long music practice and executive control in older adults: an eventrelated potential study. Brain Res. 1642, 146-153. doi: 10.1016/j.brainres.2016. 03.028

Okada, B. M., and Slevc, L. R. (2018). Individual differences in musical training and executive functions: a latent variable approach. Mem. Cogn. 46, 1076-1092. doi: 10.3758/s13421-018-0822-8

Pasiali, V., Lagasse, A. B., and Penn, S. L. (2014). The effect of musical attention control training (MACT) on attention skills of adolescents with neurodevelopmental delays: a pilot study. J. Music Ther. 51, 333-354. doi: 10.1093/jmt/thu030

Peretz, I., and Zatorre, R. (2005). Brain organization for music processing. Annu. Rev. Psychol. 56, 89-114. doi: 10.1146/annurev.psych.56.091103.070225

Pinho, A. L., de Manzano, O., Fransson, P., Eriksson, H., and Ulle, F. (2014). Connecting to create: expertise in musical improvisation is associated with increased functional connectivity between premotor and prefrontal areas. J. Neurosci. 34, 6156-6163. doi: 10.1523/JNEUROSCI.4769-13.2014

Pongan, E., Tillmann, B., Leveque, Y., and Etienne, S. (2017). Can musical or painting interventions improve chronic pain, mood, quality of life, and cognition in patients with mild alzheimer's disease? evidence from a randomized controlled trial. J. Alzheimer's Dis. 60, 663-677. doi: 10.3233/JAD170410

Rosen, D. S., Erickson, B., Kim, Y. E., Mirman, D., Hamilton, R. H., and Kounios, J. (2016). Anodal tDCS to right dorsolateral prefrontal cortex facilitates performance for novice jazz improvisers but hinders experts. Front. Hum. Neurosci. 10:579. doi: 10.3389/fnhum.2016.00579

Särkämö, T., Ripollés, P., Vepsäläinen, H., Autti, T., Silvennoinen, H. M., Salli, E., et al. (2014). Structural changes induced by daily music listening in the recovering brain after middle cerebral artery stroke: a voxel-based morphometry study. Front. Hum. Neurosci. 8:245. doi: 10.3389/fnhum.2014. 00245

Särkämö, T., Tervaniemi, M., Laitinen, S., Forsblom, A., Soinila, S., Mikkonen, M., et al. (2008). Music listening enhances cognitive recovery and mood after middle cerebral artery stroke. Brain 131(Pt 3), 866-876. doi: 10.1093/brain/ awn 013

Schaffert, N., Janzen, T. B., Mattes, K., and Thaut, M. H. (2019). A review on the relationship between sound and movement in sports and rehabilitation. Front. Psychol. 10:244. doi: 10.3389/fpsyg.2019.00244

Seinfeld, S., Figueroa, H., Ortiz-gil, J., and Sanchez-vives, M. V. (2013). Effects of music learning and piano practice on cognitive function, mood and quality of life in older adults. Front. Psychol. 4:1-13. doi: 10.3389/fpsyg.2013.00810

Shimizu, N., Umemura, T., and Matsunaga, M. (2018). Effects of movement music therapy with a percussion instrument on physical and frontal lobe function in older adults with mild cognitive impairment: a randomized controlled trial. Aging Ment. Heal. 22, 1614-1626. doi: 10.1080/13607863.2017.1379048

Slater, J., Azem, A., Nicol, T., Swedenborg, B., and Kraus, N. (2017). Variations on the theme of musical expertise: cognitive and sensory processing in percussionists, vocalists and non-musicians. Eur. J. Neurosci. 45, 952-963. doi: 10.1111/ejn. 13535

Stewart, L., Kriegstein, K., Warren, J., and Griffiths, T. D. (2006). Music and the brain: disorders of musical listening. Brain 129(Pt 10), 2533-2553. doi: 10.1093/brain/awl171

Strong, J. V., and Mast, B. T. (2019). The cognitive functioning of older adult instrumental musicians and non-musicians musicians and non-musicians. Aging Neuropsychol. Cogn. 26, 367-386. doi: 10.1080/13825585.2018.1448356

Tanaka, S., and Kirino, E. (2016). Functional connectivity of the precuneus in female university students with long-term musical training. Front. Hum. Neurosci. 10:328. doi: 10.3389/fnhum.2016.00328

Thaut, M., and Gardiner, J. (2014). "Musical attention control training," in Handbook of Neurologic Music Therapy, eds M. Thaut, and V. Hoemberg, (Oxford: Oxford University Press), 257-269.

Thaut, M., Trimarchi, P., and Parsons, L. (2014). Human brain basis of musical rhythm perception: common and distinct neural substrates for meter, tempo, and pattern. Brain Sci. 4, 428-452. doi: 10.3390/brainsci4020428

Thaut, M. H. (2005). Rhythm, Music, and the Brain: Scientific Foundtaions and Clinical Applications. New York, NY: Routledge.

Thaut, M. H., Gardiner, J. C., Holmberg, D., Horwitz, J., Kent, L., Andrews, G., et al. (2009). Neurologic music therapy improves executive function and emotional adjustment in traumatic brain injury rehabilitation. Ann. N. Y. Acad. Sci. 1169, 406-416. doi: 10.1111/j.1749-6632.2009.04585.x

Thaut, M. H., McIntosh, G. C., and Hoemberg, V. (2015). Neurobiological foundations of neurologic music therapy: rhythmic entrainment and the motor system. Front. Psychol. 5:1185. doi: 10.3389/fpsyg.2015.01185

Thaut, M. H., Peterson, D. A., and McIntosh, G. C. (2005). Temporal entrainment of cognitive functions: musical mnemonics induce brain plasticity and oscillatory synchrony in neural networks underlying memory. Ann. N. Y. Acad. Sci. 1060, 243-254. doi: 10.1196/annals.1360.017

Thompson, R. G., Moulin, C. J. A., Hayre, S., and Jones, R. W. (2006). Music enhances category fluency in healthy older adults and alzheimer 's disease patients. Exp. Aging Res. 31, 91-99. doi: 10.1080/03610730590882819

Villarreal, M. F., Cerquetti, D., Caruso, S., Schwarcz, V., and Aranguren, L. (2013). Neural correlates of musical creativity: differences between high and low creative subjects. PLoS One 8:1-11. doi: 10.1371/journal.pone.0075427

Zuk, J., Benjamin, C., Kenyon, A., and Gaab, N. (2014). Behavioral and neural correlates of executive functioning in musicians and non-musicians. PLoS One 10:e0137930. doi: 10.1371/journal.pone.0099868

Conflict of Interest: The authors declare that the research was conducted in the absence of any commercial or financial relationships that could be construed as a potential conflict of interest.

Copyright (c) 2019 Koshimori and Thaut. This is an open-access article distributed under the terms of the Creative Commons Attribution License (CC BY). The use, distribution or reproduction in other forums is permitted, provided the original author(s) and the copyright owner(s) are credited and that the original publication in this journal is cited, in accordance with accepted academic practice. No use, distribution or reproduction is permitted which does not comply with these terms. 